\title{
SPACES OF CLOSED SUBGROUPS OF A CONNECTED LIE GROUP
}

\author{
by N. OLER† \\ (Received 12 July, 1971)
}

In a sequence of two papers which appeared in 1968 and 1969 Herbert Abels [1, 2] has developed, from a method originated by Gerstenhaber [6], a means for extending the study of properly discontinuous groups of transformations to that of proper transformation groups in general. We recall that, if $G$ is a Hausdorff locally compact group of transformations of a locally compact space $X$, then the action of $G$ is proper when, for any two compact subsets $K$ and $L$, the subset $G(K, L)=\{g \in G: g L \cap K \neq 0\}$ of $G$ is compact (see [3], p. 55). In what follows all groups and spaces will be Hausdorff and locally compact. If $H$ is a closed subgroup of $G$, then it is clear that the property just defined is possessed by the action of $H$ as a group of left translations of $G$.

Let $\Sigma(G)$ be the space of closed subgroups of $G$ with the following topology. A neighbourhood $N d(H ; K, U)$ of a subgroup $H$ is determined by each compact subset $K$ of $G$ and open neighbourhood $U$ of the identity of $G$ by the definition

$$
N d(H ; K, U)=\left\{H^{\prime}: H^{\prime} \cap K \subset H U \text { and } H \cap K \subset H^{\prime} U\right\}
$$

We refer to this as the Chabauty topology, it having been defined first by Chabauty [5] for discrete subgroups. It is easy to verify (c.f. Bourbaki [4]) that the neighbourhoods defined above constitute a basis for a Hausdorff topology and that the same topology is generated if, in the above definition of $N d(H ; K, U), H U$ and $H^{\prime} U$ are replaced respectively by $U H$ and $U H^{\prime}$.

The object of this note is to apply the method of Abels to obtain a proof of the following

THEOREM. Let $G$ be a connected Lie group, $\Sigma(G)$ the Chabauty space of closed subgroups of $G$ and $\Sigma_{0}(G)$ the subspace of $\Sigma(G)$ consisting of subgroups $H$ of $G$ with the property that the quotient space $G / H$ is compact. Then $\Sigma_{0}(G)$ is open in $\Sigma(G)$.

We begin by describing the basic notion of a fundamental system as defined in [1]. (The application we wish to make does not require the more specialized " uniform fundamental system" introduced in [2].) Let $G$ be a continuous group of homeomorphisms of a space $X$. For any two subsets $K$ and $L$ of $X$, we let $G(K, L)=\{g \in G: g L \cap K \neq 0\}$ and $H(K, L)=$ $G(K, L) \cap H$ for any subset $H$ of $G$. A subset $F$ of $X$, is called a fundamental set for $G$ acting on $X$ when (i) $F$ is a $G$-covering: $G F=X$ and (ii) $G(K, F)$ is relatively compact for each compact subset $K$ of $X$. A fundamental system is a "localization" of the notion of a fundamental set obtained by restricting these requirements to suitable subsets of $X$ and $G$ respectively. More precisely, a triple $(F, Q, E)$ in which $F$ is a closed subset of $X, Q$ is an open neighbourhood of $F$ and $E$ is an open neighbourhood of the identity of $G$ is called a fundamental system (for

† This research was supported by National Science Foundation Grant GP 20138. 
$G$ acting on $X$ ) when the following conditions are satisfied:

(1) $g F \cap Q \neq 0, \forall g \in E$, i.e., $E(Q, F)=E$.

(2) If $K$ is a nonempty compact subset of $Q$, then $E(K, F)$ is nonempty and compact.

(3) For each $x \in Q$ and $g \in E(x, F)$,

(4) $E$ generates $G$.

$$
E\left(g^{-1} x, F\right)=g^{-1} E(x, F) .
$$

Condition (2) says in particular that $E F \supset Q$. Indeed, if we replace $E$ by $G$ and $Q$ by $X$, then conditions (1), (3) and (4) hold for any nonempty subset $F$ and condition (2) says precisely that $F$ is a fundamental set. The problem is in fact just this: Given a fundamental system $(F, Q, E)$, to determine conditions under which $F$ is then necessarily a fundamental set. It is shown in [1] how to construct a space $X^{\prime}$ on which $G$ acts as a continuous group of transformations and a local homeomorphism $\pi$ of $X^{\prime}$ into $X$ with the following properties. (i) $\pi$ commutes with the actions of $G$ on $X$ and $X^{\prime}$, i.e., the diagram

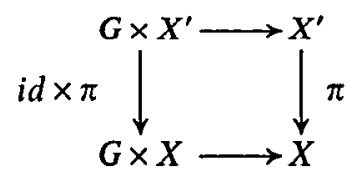

is commutative. (ii) Furthermore, $X^{\prime}$ contains an open subset $Q^{\prime}$ which is homeomorphic under $\pi$ to $Q$ and $F^{\prime}=\left.\pi\right|_{Q^{\prime}} ^{-1}(F)$ is a closed fundamental set with respect to the action of $G$ on $X^{\prime}$. The principal result concerning the " associated pair" $\left(X^{\prime}, \pi\right)$ which we shall employ is

TheOREM (H. Abels). Let $(F, Q, E)$ be a fundamental system with respect to the continuous group of transformations $G$ acting on $X$ and let $\left(X^{\prime}, \pi\right)$ be the " associated pair". If

(1) $X$ is locally connected and connected,

(2) $Q$ is connected,

(3) there exists a $G$-invariant uniform structure which induces the topology of $X$,

(4) $F$ is compact,

then $\left(X^{\prime}, \pi\right)$ is a covering space of $X$.

We proceed now to the proof of our theorem. $G$ being a connected Lie group, it is the product of a maximal compact subgroup and a euclidean space. Its fundamental group is therefore finitely generated. Furthermore, $G$ admits a simply connected covering space. It follows (see [1, Proposition 4]) that $G$ has the following property. There exists a compact subset $K$ of $G$ such that, for any covering space $\left(X^{\prime}, p\right)$ of $X$, if $X^{\prime}$ contains a subset $L$ which is homeomorphic to $K$ under $\left.p\right|_{L}$, then $\left(X^{\prime}, p\right)$ is a trivial covering space.

Let $H$ be in $\Sigma_{0}(G)$. Then there exists a compact subset $F$ of $G$ such that $H F=G$; we may further suppose that $F \supset K$ and $e \in F$. Since $G$ is locally compact, locally connected and connected, any two points of $G$ are contained in a relatively compact connected subset, from which it follows that $F$, being compact, can be covered by a finite number of relatively compact connected sets having a common point. Hence $F$ has a relatively compact connected open neighbourhood. So let $Q$ be such a neighbourhood of $F$ and let $E=H(Q, F)$. Then $E$ 
is a relatively compact subset containing the identity which is open in $H$. We choose $W$, a compact neighbourhood of the identity, such that $W F \subset Q$ and consider the neighbourhood $N d(H, \bar{E}, W)$ of $H$ in $\Sigma(G)$. Let $H^{\prime} \in N d(H, \bar{E}, W)$; then $H^{\prime} \cap \bar{E} \subset H W$ and $H \cap \bar{E} \subset H^{\prime} W$. If $q \in Q$, then $q=g f$ for some $g \in E$ and $f \in F$. Also, $g \in E$ implies that $g=g^{\prime} w$ for some $g^{\prime} \in H^{\prime}$ and $w \in W$. Thus $q=g^{\prime} w f$ and $Q \subset H^{\prime} W F$. Set $E^{\prime}=H^{\prime}(Q, W F)$; then clearly $E^{\prime}=$ $E^{\prime}(Q, W F)$ and $Q \subset E^{\prime} W F$. Let $K$ be any nonempty compact subset of $Q$. Then $E^{\prime}(K, W F)$ is nonempty and furthermore $E^{\prime}(K, W F)=K F^{-1} W^{-1} \cap H^{\prime}$, a closed subset of $K F^{-1} W^{-1}$ which is compact; hence $E^{\prime}(K, W F)$ is compact. Let $x \in Q$ and $g \in E^{\prime}(x, W F)$. Since $H^{\prime}$ is a subgroup, it is clear that $g^{-1} x F^{-1} W^{-1} \cap H^{\prime}=g^{-1}\left[x F^{-1} W^{-1} \cap H^{\prime}\right]$ and hence $E^{\prime}\left(g^{-1} x, W F\right)=g^{-1} E^{\prime}(x, W F)$. Now let $H^{\prime \prime}$ be the subgroup of $H^{\prime}$ generated by $E^{\prime}$. What we have shown is that $\left(W F, Q, E^{\prime}\right)$ is a fundamental system for $H^{\prime \prime}$ acting on $G$. The conditions of Abels's theorem are satisfied, enabling us to conclude therefore that $\left(G^{\prime}, \pi\right)$, the associated space of $\left(W F, Q, E^{\prime}\right)$, is a covering space of $G$. We have moreover that $G^{\prime}$ contains a subset $F^{\prime}$ which is homeomorphic to $W F$ under $\left.\pi\right|_{F^{\prime}}$. It follows that $\pi$ must therefore be a homeomorphism. Since, furthermore, $F^{\prime}$ is a fundamental set and $\pi$ commutes with the actions of $H^{\prime \prime}$ on $G^{\prime}$ and $G$, we find that $F$ is a fundamental set for $H^{\prime \prime}$ acting on $G$, so that, in particular, $H^{\prime \prime} W F=G$. Now, for $g \in H^{\prime}, g=g_{1} f$, where $g_{1} \in H^{\prime \prime}$ and $f \in W F$. This implies that $g^{-1} g_{1} \in H^{\prime}(e, W F) \subset H^{\prime}(Q, W F)=E^{\prime}$; hence $g^{-1} \in g_{1}^{-1} E^{\prime} \subset H^{\prime \prime}$. Thus $H^{\prime}=H^{\prime \prime}$ and the proof is complete.

We conclude by remarking that, if $S(G)$ and $S_{0}(G)$ denote the subspaces of $\Sigma(G)$ and $\Sigma_{0}(G)$, respectively, obtained by restricting to discrete subgroups, then A. M. Macbeath [7] has shown that $S_{0}(G)$ is the union of open subsets of $S(G)$ consisting of isomorphic subgroups.

\section{REFERENCES}

1. H. Abels, Über die Erzengung von eigentlichen Transformationsgruppen, Math. Zeit. 103 (1968), 333-357.

2. H. Abels, Úber eigentliche Transformationsgruppen, Math. Zeit. 110 (1969), 75-100.

3. N. Bourbaki, Eléments de Mathématique, $3^{\mathrm{e}}$ édn., Topologie Générale, Chap. 3, Groupes Topologiques (Paris, 1961).

4. N. Bourbaki, Éléments de Mathématique, Intégration, Chap. 8, Convolution et Représentations, (Paris, 1963).

5. C. Chabauty, Limites d'ensembles et géometrie des nombres, Bull. Soc. Math. France 78 (1950), 143-151.

6. M. Gerstenhaber, On the algebraic structure of discontinuous groups, Proc. Amer. Math. Soc. 4 (1953), 745-750.

7. A. M. Macbeath, Groups of homeomorphisms of a simply connected space, Ann. of Math. 79 (1964), 473-488.

\section{University of Pennsylvania \\ and}

UNIVERSITY OF BIRMINGHAM 